\title{
Adiabatic matching section for plasma accelerated beams
}

\author{
Klaus Floettmann \\ DESY, Notkestrasse 85, 22603 Hamburg, Germany \\ (Received 10 February 2014; published 7 May 2014)
}

\begin{abstract}
An adiabatic matching section is discussed as option to control the divergence and emittance growth of a beam exiting a plasma channel. Based on a general analytical solution of a focusing channel with varying focusing strength, a focusing profile is proposed which allows for a fast expansion of the beam size while keeping the emittance growth minimal. The solution is also applicable to other cases, e.g., the matching of a positron source to the downstream accelerating section, which are, however, not discussed in this contribution.
\end{abstract}

DOI: 10.1103/PhysRevSTAB.17.054402

PACS numbers: 29.27.-a, 41.85.-p, 41.75.Fr

\section{INTRODUCTION}

Plasma waves are considered as prime candidates for novel compact accelerators for their ability to support extreme accelerating fields in the $\mathrm{GeV}$ per centimeter scale. Compared to conventional rf structure based accelerators not only the accelerating field strength but also the short wavelength of some tens to some hundreds of micrometers, the small transverse dimensions of similar order and the strong focusing fields inside the plasma channel are extreme. Besides technical challenges plasma waves are therefore also challenging from a beam dynamics point of view.

In a recent paper [1] the matching conditions which need to be fulfilled in order to avoid emittance growth due to betatron phase mixing in a plasma channel have been discussed. This study is extended here to the transition from the plasma to a "free" beam, i.e., a beam outside of the plasma channel. Transitions are necessary whenever the beam shall be used outside of the plasma, e.g., to send it through an undulator, but also when a beam is injected into a plasma channel as in staged accelerator concepts. The beam at the exit of a plasma channel is characterized by a small beam spot and a large beam divergence, often in combination with a large energy spread, while for a conventional beam transport a larger beam size and a small beam divergence are required. Thus a matching section in between these quite different optical sections is required.

Equivalent conditions are found, e.g., at the source point of a positron source where the positron beam exits the target. Helm [2] has discussed the beam dynamics in a tapered solenoid field which can be used to match a positron beam to the downstream accelerating section.

"Klaus.Floettmann@DESY.De

Published by the American Physical Society under the terms of the Creative Commons Attribution 3.0 License. Further distribution of this work must maintain attribution to the author $(s)$ and the published article's title, journal citation, and DOI.
Helm's solution is approximate and valid for adiabatic variations of the focusing strength. In this contribution a new, exact analytic solution is presented which is valid for a particular functional dependence of the variation of the focusing strength. The exact solution converges to the adiabatic solution; a comparison with Helm's approximation is presented in the appendix. Numerical simulations show that the beam divergence and the emittance growth can be controlled with an adiabatic matching section. The new field profile of the analytical solution is found to be superior to the profile proposed by Helm. The discussion concentrates on the transition from plasma to vacuum but the results can equally be used to match from the vacuum into a plasma channel.

\section{THE CONDITIONS INSIDE THE PLASMA CHANNEL}

Before discussing the transition section the conditions inside the plasma channel as presented in [1] shall be summarized. The plasma wave presents a focusing channel to particles traveling through the plasma at the speed of the plasma wave. The transverse force depends on the longitudinal position inside the wave, but to first order not on the position of the wave inside the plasma. Thus slices of particles inside the bunch are subject to a constant focusing force while traversing the plasma channel. (Adiabatic variations of the force will not change the results as discussed below.) Matched conditions are reached when the focusing compensates the expansion of the beam due to the beam emittance, i.e., when the beam follows a kind of Brillouin flow. With the usual definitions of the Courant Snyder parameters:

$\beta=\frac{\left\langle x^{2}\right\rangle}{\varepsilon}, \quad \alpha=-\frac{\left\langle x x^{\prime}\right\rangle}{\varepsilon}, \quad \gamma=\frac{\left\langle x^{\prime 2}\right\rangle}{\varepsilon}, \quad \beta \gamma=1+\alpha^{2}$,

where $\varepsilon$ represents the transverse rms trace-space emittance and \langle\rangle denotes the central average value of the variable in the brackets, the matching condition yields $\alpha=0$ and 
thus $\gamma=1 / \beta$. Furthermore, the beta function in a focusing channel with constant focusing strength $K$ writes for $\alpha_{0}=0$ (index zero indicates initial conditions) as:

$$
\beta=\beta_{0} \cos ^{2} \sqrt{K} z+\frac{1}{\beta_{0} K} \sin ^{2} \sqrt{K} z
$$

which is constant if the relation $K=1 / \beta_{0}^{2}$ is fulfilled. If a beam is not matched, the beta function oscillates. Since the focusing strength in a wave depends in a sinelike manner on the relative longitudinal position within the wave, different longitudinal slices oscillate with different frequencies, so that the projected emittance increases until the matching condition is reached for the projected phasespace. In case of a pure mismatch of the beta function (i.e., $\alpha_{0}=0$ holds) the ratio of the final emittance $\varepsilon_{f}$ to the initial emittance $\varepsilon_{i}$ is given as [1]:

$$
\frac{\varepsilon_{f}}{\varepsilon_{i}}=\frac{1}{2} \frac{\beta_{m}^{2}+\beta_{0}^{2}}{\beta_{m} \beta_{0}}
$$

where $\beta_{m}$ stands for the matched beta function. Equation (3) is equally valid for a beam which is injected into a plasma channel as for a beam which is generated inside the channel. Especially in case of self-injection the captured electrons form a beam with large energy spread. Since the focusing strength is inversely proportional to the particle momentum, the matched beta function differs for the different energies in the bunch which leads to an additional contribution to the emittance in accordance to Eq. (3). The phase-space mixing is a fast process; we can thus assume that a beam obeys the matching conditions when it reaches the end of the plasma channel.

A particular problem encountered in the case of selfinjected beams is related to the fact that the canonical phase-space emittance grows strongly in a drift in all cases where a large beam divergence is combined with a large energy spread. This fundamental process is described in general form in [3] and applied to the case of a plasma based electron source in [4]. The relevance of the phasespace emittance lies in the fact that the trace-space emittance rapidly growths up to the value of the phasespace emittance as soon as the divergence of the beam is reduced, i.e., in the next focusing element [3].

A reduction of the divergence of a plasma accelerated beam by an appropriately designed transition region from plasma to vacuum in which the beam size is increased is considered in [5]. The emittance measurements presented therein show the trace-space emittance. A conclusion on the phase-space emittance can, despite the somewhat reduced divergence, not be drawn since the divergence is still high. A reliable emittance measurement can in the case of self-injected beams only be performed behind a focusing element.

The analytical treatment of a matching section presented below concentrates on the optical functions and thus on the trace-space emittance. Distortions of the phase-space emittance are addressed by numerical simulations.

\section{THE MATCHING SECTION}

In the matching section the focusing strength $K(z)$ provided by the plasma wave shall be decreased in such a way that the beam size expands in a controlled way and the beam divergence in the following drift is reduced. Noting that the equation of motion of individual particles needs to converge to the known case of a constant focusing channel if the focusing strength converges to a constant, the following ansatz is formulated:

$$
x=A(z) \cos \int \sqrt{K(z)} d z+\frac{B(z)}{\sqrt{K(z)}} \sin \int \sqrt{K(z)} d z .
$$

$A(z)$ and $B(z)$ are arbitrary functions.

The single particle differential equation $x^{\prime \prime}+K x=0$ can be solved for this case (see Appendix A.1 for details) and yields:

$$
x=x_{0}(1+g z) \cos \varphi+\frac{\left(x_{0}^{\prime}-x_{0} g\right)(1+g z)}{\sqrt{K_{0}}} \sin \varphi,
$$

where the following functional dependence of the focusing strength is assumed:

$$
K(z)=\frac{K_{0}}{(1+g z)^{4}} \quad g=-\frac{K_{0}^{\prime}}{4 K_{0}} .
$$

$g$ is a taper parameter introduced to describe the decay of the focusing field and $K_{0}$ and $K_{0}^{\prime}$ are the initial focusing strength and the first derivative of the focusing strength with respect to the longitudinal coordinate $z$. The phase advance $\varphi$ is found as:

$$
\varphi=\frac{\sqrt{K_{0}} z}{1+g z},
$$

which converges for large $z$ to $\varphi_{\infty}=\frac{\sqrt{K_{0}}}{g}$. Figure 1 shows some example trajectories for different values of $\varphi_{\infty}$. It illustrates the transition from a free expansion for small phase advance to the case of an adiabatic expansion with an oscillation of slowly increasing amplitude for larger phase advance.

Equation (5) describes the motion of individual particles. To describe the envelope of a beam, Eq. (5) needs to be squared followed by averaging over the variables. After replacements according to Eq. (1) the beta function follows as: 


$$
\beta=(1+g z)^{2}\left[\beta_{0}\left(\cos ^{2} \varphi-\frac{2 g}{\sqrt{K_{0}}} \cos \varphi \sin \varphi+\frac{g^{2}}{K_{0}} \sin ^{2} \varphi\right)-\alpha_{0}\left(\frac{2}{\sqrt{K_{0}}} \cos \varphi \sin \varphi-\frac{2 g}{K_{0}} \sin ^{2} \varphi\right)+\gamma_{0}\left(\frac{1}{K_{0}} \sin ^{2} \varphi\right)\right]
$$

This equation simplifies greatly when the matching conditions for the beam in the plasma are introduced. With $\alpha_{0}=0, \gamma_{0}=\frac{1}{\beta_{0}}, K_{0}=1 / \beta_{0}^{2}$ we get:

$$
\begin{aligned}
\beta & =\beta_{0}(1+g z)^{2}\left[\left(\cos \varphi-g \beta_{0} \sin \varphi\right)^{2}+\sin ^{2} \varphi\right] \\
& \approx \beta_{0}(1+g z)^{2}=\beta_{0}\left[\frac{K_{0}}{K(z)}\right]^{\frac{1}{2}}
\end{aligned}
$$

The approximation made in the second step requires that $g \beta_{0} \ll 1$. The same approximation is made by Helm in his derivation of the adiabatic approximation (see Appendix A.2 for details). The connection to the adiabatic solution becomes obvious by noting that $g \beta_{0}=\varphi_{\infty}^{-1}$, i.e., $g \beta_{0} \ll 1$ corresponds to the case of a large phase advance as expected for an adiabatic behavior. While the exact solution is valid only for a tapered focusing field in accordance to Eq. (6), the adiabatic solution is valid for arbitrary spatial dependencies of the focusing strength provided that the variation of the focusing strength is slow as compared to the phase advance.

The other extreme of Eq. (9) is found for a small phase advance by setting $\cos \varphi_{\infty}=1, \sin \varphi_{\infty}=\sqrt{K_{0}} / g$ which yields $\beta \approx \frac{z^{2}}{\beta_{0}}$. This is identical to the result of a beam in a free drift which starts with $\beta=\beta_{0}$ at the exit of the plasma channel. Of great practical importance is the transition between adiabatic and nonadiabatic motion. The slightly nonadiabatic case marks conditions where the fastest expansion of the beam size is realized and nonadiabatic effects just start to play a role. Since in a general case the adiabaticity can differ for different locations of the field profile, the question of the optimal field profile is directly connected with the slightly nonadiabatic case. Both in the fully adiabatic and in the fully nonadiabatic case the details of the field profile are of minor importance.

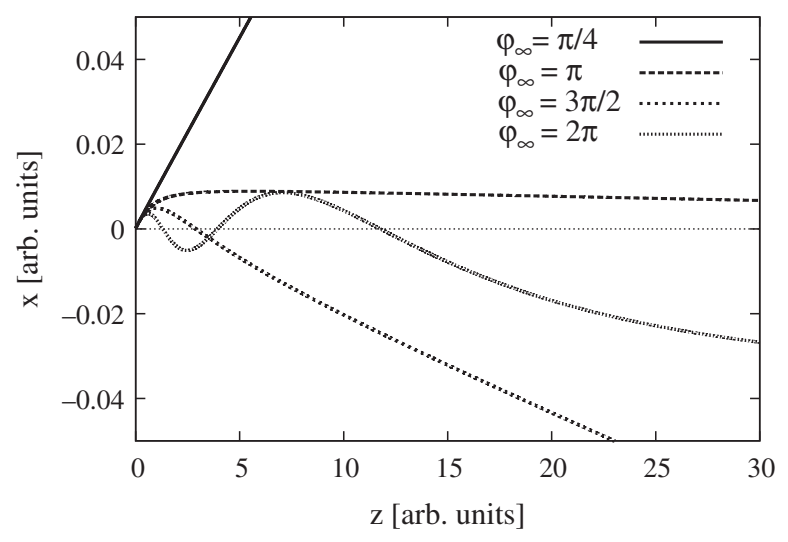

FIG. 1. Example trajectories for various values of the phase advance.
Equation (9) is independent of the particle energy, i.e., the trace-space emittance is conserved not only in the adiabatic limit, but also in the free space limit. However, in order to keep the growth of the canonical phase-space emittance under control it is important to keep the divergence of the beam small. From the relation $\alpha=-\frac{1}{2} \beta^{\prime}$ we find in the free space limit:

$$
\alpha_{f} \approx-\frac{z}{\beta_{0}}
$$

while it is reduced in the adiabatic case to:

$$
\alpha_{a}=-\beta_{0} g(1+g z) \approx-\beta_{0} g^{2} z
$$

The ratio is thus just given by the inverse square of the total phase advance:

$$
\frac{\alpha_{a}}{\alpha_{f}}=\left(\beta_{0} g\right)^{2}=\varphi_{\infty}^{-2}
$$

For a comparison of the emittance growth it is, however, not reasonable to compare parameters at the same longitudinal position, because in the case of an adiabatic matching section a much longer distance is required to expand the beam by a significant amount.

When the matching section ends the beam expands in a free drift with the initial conditions $\beta_{e}$ and $\alpha_{e}$ which mark the optical functions at the end of the matching section. The derivative of the expansion in the subsequent drift is given by:

$$
\beta_{\mathrm{drift}}^{\prime}=-2 \alpha_{e}+2\left(\frac{1+\alpha_{e}^{2}}{\beta_{e}}\right) \Delta z
$$

where $\Delta z$ is the distance relative to the end of the matching section. Equation (13) is dominated by $\alpha_{e}$ when $\alpha_{e}^{2}>1$. In this case the beam continues to expand in the drift just as in the matching section. Thus the maximum reasonable length of the matching section is defined by the condition $\alpha_{e}^{2}>1$.

\section{NUMERICAL CALCULATIONS}

For the following numerical examples beam parameters as used in Ref. [4] have been chosen to allow a comparison. The parameters are summarized in Table I; the focusing strength is tapered according to Eq. (6). The simulation assumes a theoretical radial focusing force which does not depend on the position within the bunch. Longitudinal forces as they appear in a plasma channel but also in other focusing elements are not taken into account. 
TABLE I. Main simulation parameters.

\begin{tabular}{ll}
\hline \hline Normalized emittance & $2.5 \times 10^{-6} \mathrm{~m}$ \\
Energy & $912 \mathrm{MeV}$ \\
rms energy spread & $6.4 \%$ \\
Initial beam size & $0.5 \times 10^{-6} \mathrm{~m}$ \\
$\beta_{0}$ & $1.8 \times 10^{-4} \mathrm{~m}$ \\
$K_{0}$ & $3.14 \times 10^{7} \mathrm{~m}^{-2}$ \\
\hline \hline
\end{tabular}

Figure 2 compares the development of the beam size in a matching section for varying taper parameter $g$. The beam size and divergence can be controlled as predicted by theory. The factor $g \beta_{0}$ yields values from 0.04 for $g=$ $200 \mathrm{~m}^{-1}$ up to 0.12 for $g=700 \mathrm{~m}^{-1}$ and thus spans a parameter range from the fully adiabatic expansion into a slightly nonadiabatic expansion. This is reflected in the development of the normalized phase-space emittance which is plotted in Fig. 3 for the four cases shown in

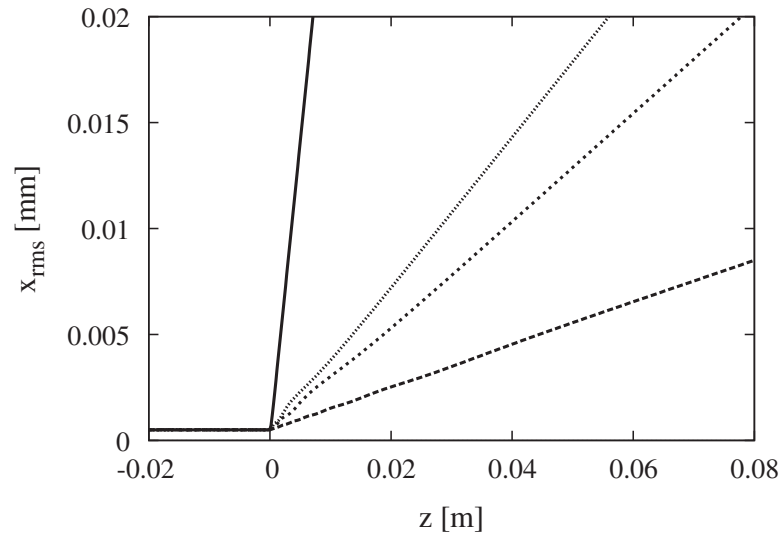

FIG. 2. Development of the beam size behind a plasma channel for $g=200 \mathrm{~m}^{-1}$ (smallest divergence), $500 \mathrm{~m}^{-1}, 700 \mathrm{~m}^{-1}$ and the free space solution (largest divergence). The constant focusing channel ends at $z=0$.

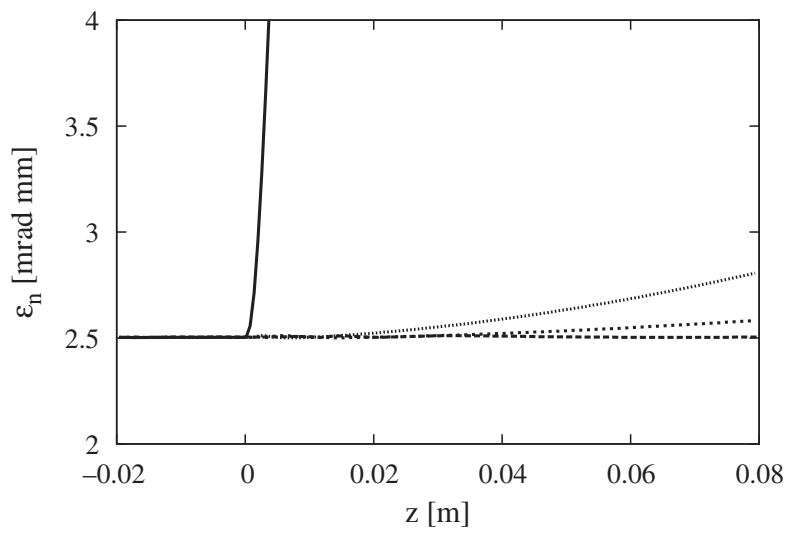

FIG. 3. Development of the normalized phase-space emittance behind a plasma channel. Parameters and line styles correspond to Fig. 2. The trace-space emittance (not shown) stays constant in all cases.
TABLE II. Comparison of beam parameters at equal transverse size of $0.02 \mathrm{~mm}$ corresponding to a beam expansion by a factor of 40 .

\begin{tabular}{lcc}
\hline \hline $\begin{array}{c}\text { Taper parameter } g \\
\mathrm{~m}^{-1}\end{array}$ & $\begin{array}{c}\text { Longitudinal position } \\
10^{-2} \mathrm{~m}\end{array}$ & $\begin{array}{c}\text { Emittance } \\
10^{-6} \mathrm{~m}\end{array}$ \\
\hline 200 & 21.6 & 2.53 \\
500 & 7.8 & 2.58 \\
700 & 5.6 & 2.65 \\
Free space & 0.7 & 6.9 \\
\hline \hline
\end{tabular}

Fig. 2 with corresponding line styles. While the trace-space emittance (not shown) stays constant in all cases the phasespace emittance increases when the beam expands. The emittance growth is dramatic in case of a free drift, but negligible in case of a fully adiabatic expansion. Table II compares the phase-space emittance which is reached at equal transverse beam size.

The emittance growth due to the nonadiabaticity of the field can be treated as a quadratic addition to the incoming emittance. This contribution is then found to scale linearly with the energy spread of the beam. A reduced energy spread thus allows us to choose parameters deeper in the nonadiabatic region, i.e., to realize a faster expansion of the beam without emittance growth.

In Fig. 4 the further development in a drift following the matching section is illustrated. As discussed in the previous paragraph the beam continues to expand in the drift just as in the matching section when the condition $\alpha_{e}^{2}>1$ is fulfilled.

\section{BEST FIELD PROFILE}

In the adiabatic approximation the beta function just scales as the square root of the initial to the local focusing strength $\beta(z)=\beta_{0}\left[\frac{K_{0}}{K(z)}\right]^{\frac{1}{2}}$ and is in this form applicable to

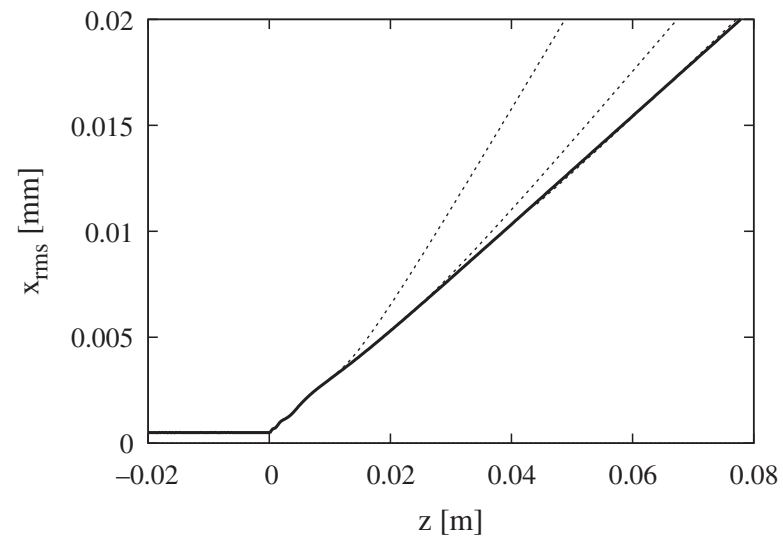

FIG. 4. Development of the transverse beam size in a matching section with $g=500 \mathrm{~m}^{-1}$ (solid line). Broken lines indicate the further development in a drift when the matching section ends at $\left|\alpha_{e}\right|=0.5,1.0$ and $\left|\alpha_{e}\right|=2.0$. The last line overlaps already completely with the solid line. 
any variation of the focusing strength provided that the variation is adiabatic. This opens the question of which functional dependence of the focusing strength is optimal in the sense that it yields the fastest expansion of the beam with minimal emittance growth. In fact Helm formulated the adiabatic solution without assumption of a specific functional dependence and proposed in a second step only a focusing following:

$$
K(z)=\frac{K_{0}}{\left(1+g_{H} z\right)^{2}},
$$

as ideal field profile. From the definition of the adiabaticity parameter one concludes that Helm's taper parameter $g_{H}=2 g$. In this case the weaker power of Helm's scaling law leads to a much smaller beam size at the end of the matching section. For a better comparison comparable beam sizes at the end of the section are required. Since we are here interested in the slightly nonadiabatic case this requires some tuning of the taper parameter. Figure 5 compares the beam size development for the power of four scaling [cf. Eq. (6)] as found in the analytical treatment with $g=700 \mathrm{~m}^{-1}$ with the power of two scaling as proposed by Helm with $g_{H}=9000 \mathrm{~m}^{-1}$. Despite the comparable beam size at the end of the section the emittance, shown in Fig. 6, is significantly larger in case of Helm's field profile. This is due to the fact that a fast expansion in the beginning of the matching section is required in order to reach the desired beam size because the expansion is strongly reduced in the last part of the section. The rapid expansion of the beam size leads to a strong increase of the emittance which outranges the emittance growth of the other field profile with the linearly growing beam size. Due to the reduction of the beam divergence in the last part of the section the emittance growth in phase-space now also shows up as equivalent growth of the trace-space emittance. The new profile found in this study is thus the better solution for the matching of beams with large divergence and energy spread when the

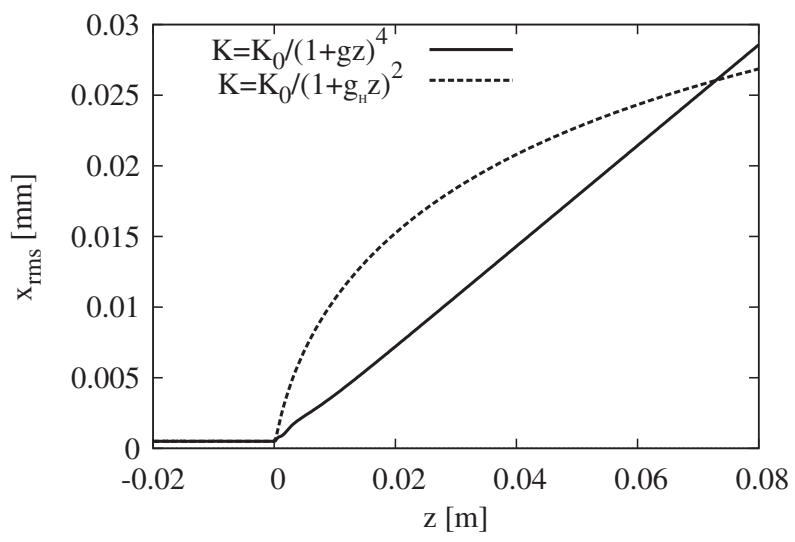

FIG. 5. Development of the beam size for different field profiles.

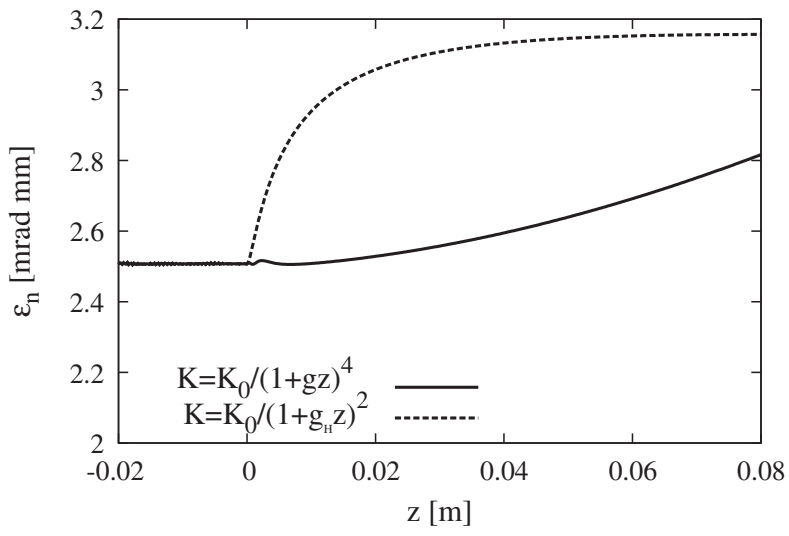

FIG. 6. Development of the transverse emittance for the field profiles shown in Fig. 4.

emittance growth shall be minimized. (If emittance growth is not a concern, Helm's solution offers a better control over the beam divergence.) This finding does not prove that a focusing profile following Eq. (6) is the optimum of all possible field profiles but supports the assumption that this is the case. Indeed, if we understand adiabaticity as a condition where a variation of the focusing develops so slowly that the beam size can follow the variation, a channel in which the beam size expands at a constant rate appears to be optimal.

\section{CONCLUSION}

Adiabatic matching sections are an option to control the beam divergence and the emittance growth of beams exiting a plasma channel. Future investigations need to be based on realistic density profiles of a plasma down-ramp and take into account effects as phase dependent forces, phase slippage, damping of the beam divergence, and energy variation due to the longitudinal field components. Additional variations of the focusing forces may appear as a result of the divergence of a plasma driving laser or charged particle beam. While in the latter case only the amplitude of the wave changes, the plasma wavelength changes in addition in case of density variations, which is associated with phase shifts. The discussion above suggests that we seek a density profile in which the beam expands at a constant rate to meet a constant adiabaticity condition at all locations even in cases where the theory developed in this presentation is not strictly applicable. The dynamics in a plasma of varying density is complicated and it is not a priori clear that appropriate solutions for the adiabatic matching section can be found for all plasma parameters without being hampered by detrimental effects like deceleration due to phase shifts. The analytical solution can serve as a guideline for the optimization and help to disentangle effects of fundamental nature from effects which are related to the specific dynamics in a plasma channel. In the case of beams with large energy spread 
relatively long matching sections are required to be effective, which might also not be practical in all cases. A further reduction of the energy spread is hence highly desirable.

\section{ACKNOWLEDGMENTS}

Thanks to A. R. Maier (UHH/CFEL) and I. Dornmair (UHH/CFEL) for stimulating discussions and useful suggestions.

\section{APPENDIX}

\section{Solution of the differential equation}

Following the ansatz:

$$
x=A(z) \cos \int \sqrt{K(z)} d z+\frac{B(z)}{\sqrt{K(z)}} \sin \int \sqrt{K(z)} d z
$$

the single particle differential equation $x^{\prime \prime}+K x=0$ is written as:

$$
\begin{aligned}
&-\left(2 \sqrt{K} A^{\prime}+\frac{A K^{\prime}}{2 \sqrt{K}}\right) S+A^{\prime \prime} C+\left(2 B^{\prime}-\frac{B K^{\prime}}{2 K}\right) C \\
&+\left(-\frac{B^{\prime} K^{\prime}}{K^{3 / 2}}+\frac{3 B K^{\prime 2}}{4 K^{5 / 2}}+\frac{B^{\prime \prime}}{\sqrt{K}}-\frac{B K^{\prime \prime}}{2 K^{3 / 2}}\right) S=0, \\
& C:=\cos \int \sqrt{K} d z, \quad S:=\sin \int \sqrt{K} d z .
\end{aligned}
$$

This equation can only be fulfilled if individual terms yield zero. Thus from:

$$
\left(2 \sqrt{K} A^{\prime}+\frac{A K^{\prime}}{2 \sqrt{K}}\right)=0, \quad\left(2 B^{\prime}-\frac{B K^{\prime}}{2 K}\right)=0,
$$

we get:

$$
\frac{A}{A^{\prime}}=-\frac{4 K}{K^{\prime}}, \quad \frac{B}{B^{\prime}}=\frac{4 K}{K^{\prime}} .
$$

Moreover,

$$
A^{\prime \prime}=\frac{A^{\prime} K^{\prime}}{4 K}+\frac{A K^{\prime 2}}{4 K^{2}}-\frac{A K^{\prime \prime}}{4 K}=\left(-\frac{5}{4} \frac{K^{\prime}}{K}+\frac{K^{\prime \prime}}{K^{\prime}}\right) A^{\prime}=0
$$

and

$$
B^{\prime \prime}=\left(-\frac{3}{4} \frac{K^{\prime}}{K}+\frac{K^{\prime \prime}}{K^{\prime}}\right) B^{\prime}
$$

$$
\left(-\frac{B^{\prime} K^{\prime}}{K^{3 / 2}}+\frac{3 B K^{\prime 2}}{4 K^{5 / 2}}+\frac{B^{\prime \prime}}{\sqrt{K}}-\frac{B K^{\prime \prime}}{2 K^{3 / 2}}\right)=-\left(-\frac{5 K^{\prime}}{4}+\frac{K^{\prime \prime}}{K^{\prime}}\right) \frac{B^{\prime}}{\sqrt{K}}=0 .
$$

Hence, in order to fulfill Eq. (A2) the condition:

$$
-\frac{5}{4} \frac{K^{\prime}}{K}+\frac{K^{\prime \prime}}{K^{\prime}}=0
$$

needs to be fulfilled, with the solution:

$$
K(z)=\frac{K_{0}}{\left(1-\frac{K_{0}^{\prime}}{4 K_{0}} z\right)^{4}}
$$

and

$$
K^{\prime}(z)=K(z) \frac{K_{0}^{\prime}}{K_{0}\left(1-\frac{K_{0}^{\prime}}{4 K_{0}} z\right)}, \quad \int \sqrt{K} d z=\frac{\sqrt{K_{0}} z}{1-\frac{K_{0}^{\prime}}{4 K_{0}} z}
$$

where an integration constant is chosen such that the integral is zero for $z=0$.

From $A(0) \cos \int \sqrt{K} d z=A(0)$ follows $A(0)=x_{0}$. Introducing $K$ and $K^{\prime}$ into $A=-4 \frac{K}{K^{\prime}} A^{\prime}$ yields $A=$ $-4 C\left(1-\frac{K_{0}^{\prime}}{4 K_{0}} z\right)$ with the integration constant $C$. Thus with $C=-x_{0} / 4$ we get:

$$
A(z)=x_{0}\left(1-\frac{K_{0}^{\prime}}{4 K_{0}} z\right) .
$$

Equivalently follows from $B=4 \frac{K}{K^{\prime}} B^{\prime} B=\frac{C}{4}\left(\frac{1}{1-\frac{K_{0}^{\prime}}{4 K_{0} z}}\right)$ and thus with $x^{\prime}(0)=x_{0}^{\prime} C=4\left(x_{0}^{\prime}-A^{\prime}\right)$ which finally yields:

$$
B(z)=\frac{x_{0}^{\prime}-A^{\prime}}{1-\frac{K_{0}^{\prime}}{4 K_{0}} z} .
$$

Introducing the taper parameter $g$ and the phase advance $\varphi$ yields Eq. (5).

\section{Comparison with Helm's adiabatic approximation}

Helm [2] formulated an approximate solution for a tapered solenoid field. The approximation is valid if the adiabaticity parameter $\varepsilon=\left|\frac{B^{\prime} p_{z}}{e B^{2}}\right| \ll 1$. This condition is equally fulfilled for all positions $z$ if the field follows the condition:

$$
B=\frac{B_{0}}{1+g_{H} z},
$$

where $g_{H}$ is a taper parameter and $B_{0}$ is the initial solenoid field value. 
The adiabaticity parameter writes then as:

$$
\varepsilon=\frac{g_{H} p_{z}}{e B_{0}} \ll 1
$$

The focusing strength of a solenoid is given by $K=\left(\frac{e B}{2 p_{z}}\right)^{2}$. It follows:

$$
K(z)=\frac{K_{0}}{\left(1+g_{H} z\right)^{2}}, \quad K^{\prime}(z)=\frac{-2 g_{H} K_{0}}{\left(1+g_{H} z\right)}
$$

with the initial focusing strength $K_{0}=\left(\frac{e B_{0}}{2 p_{z}}\right)^{2}$ and $K_{0}^{\prime}=-2 g_{H} K_{0}$. Thus the taper parameter $g_{H}$ can be replaced in Eq. (A15) to yield:

$$
K(z)=\frac{K_{0}}{\left(1-\frac{K_{0}^{\prime}}{2 K_{0}} z\right)^{2}}=\frac{K_{0}}{1-\frac{K_{0}^{\prime}}{K_{0}} z+\left(\frac{K_{0}^{\prime}}{K_{0}}\right)^{2} \frac{z}{4}^{2}}
$$

which needs to be compared to:

$$
K(z)=\frac{K_{0}}{\left(1-\frac{K_{0}^{\prime}}{4 K_{0}} z\right)^{4}}=\frac{K_{0}}{1-\frac{K_{0}^{\prime}}{K_{0}} z+\left(\frac{K_{0}^{\prime}}{K_{0}}\right)^{2} \frac{3 z^{2}}{8}-\left(\frac{K_{0}^{\prime}}{K_{0}}\right)^{3} \frac{z^{3}}{16}+\left(\frac{K_{0}^{\prime}}{K_{0}}\right)^{4} \frac{z^{4}}{256}} .
$$

Helm's definition of the taper parameter $g_{H}$ hence differs by a factor of two from the definition introduced in this paper. The resulting field profile is identical for small values of $z$ but differs for large $z$.

The adiabaticity parameter

$$
\varepsilon=\frac{g_{H} p_{z}}{e B_{0}}=\frac{g_{H}}{2 \sqrt{K_{0}}}=\varphi_{\infty}^{-1}
$$

is identical to the inverse phase advance in the limit of large $z$ of the analytical solution presented in this paper. With $B_{0} / B=1+g_{H} z, \frac{4 p_{z}}{B_{0} B}=\frac{1}{\sqrt{K_{0} K}}=\frac{1+g_{H} z}{K_{0}}$ and $\int \frac{e B}{2 p_{z}} d z=\int \sqrt{K} d z=\varphi$ Helm's solution for the particle radius which takes over the role of the transverse coordinate in the rotating Larmor frame writes as:

$$
r=r_{0}\left(1+g_{H} z\right)^{\frac{1}{2}} \cos \varphi+\frac{r_{0}^{\prime}\left(1+g_{H} z\right)^{\frac{1}{2}}}{\sqrt{K_{0}}} \sin \varphi,
$$

$\left(1+g_{H} z\right)^{\frac{1}{2}}=\left[\frac{K_{0}}{K(z)}\right]^{\frac{1}{4}}=1+g z$

which needs to be compared to Eq. (5). The addition of $-x_{0} g$ in the second term of Eq. (5) leads to the additional term in the envelope equation (9) which is neglected in case of the adiabatic approximation.

[1] T. Mehrling, J. Grebenyuk, F. S. Tsung, K. Floettmann, and J. Osterhoff, Phys. Rev. ST Accel. Beams 15, 111303 (2012).

[2] R. H. Helm, Report No. SLAC-4, 1962, http://www.slac .stanford.edu/pubs/slacreports/slac-r-004.html.

[3] K. Floettmann, Phys. Rev. ST Accel. Beams 6, 034202 (2003).

[4] M. Migliorati, A. Bacci, C. Benedetti, E. Chiadroni, M. Ferrario, A. Mostacci, L. Palumbo, A. Rossi, L. Serafini, and P. Antici, Phys. Rev. ST Accel. Beams 16, 011302 (2013).

[5] Ch. Sears, A. Buck, K. Schmid, J. Mikhailova, F. Krausz, and L. Veisz, Phys. Rev. ST Accel. Beams 13, 092803 (2010). 